\title{
The Effect of Trimethoprim-Polymyxin B Sulphate Ophthalmic Ointment and Chloramphenicol Ophthal- mic Ointment on the Bacterial Flora of the Eye when Administered to the Operated and Unoperated Eyes of Patients Undergoing Cataract Surgery
}

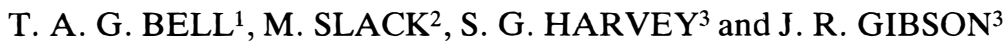 \\ Oxford and Beckenham
}

\begin{abstract}
Summary
Both eyes of patients undergoing cataract surgery were treated with an ointment preparation containing either trimethoprim $5 \mathrm{mg} / \mathrm{g}$ and polymyxin B sulphate 10,000 units/g, or chloramphenicol $1 \%$. The antibiotic preparations were administered four times daily on the day prior to surgery, once in the morning prior to surgery and twice daily for fourteen days post-operatively. Cultures from conjunctivae and lid margins were taken prior to treatment, pre-operatively and post-operatively on selected days including the fourteenth post-operative day.

The effect of the antibiotics on conjunctival and lid margin flora was determined by enumerating patients with positive cultures prior to treatment which were eradicated following treatment and patients with organisms which were cultured de novo in spite of antibiotic treatment.

There was no significant change in the number of patients with organisms isolated from conjunctivae and lid margins ( $p>0.1$ in each case, Sign test) over the sixteen day study period with either treatment. Additionally, when the effect of the two preparations on the number of patients with organisms obtained from conjunctivae and lid margins was compared, there was no significant difference between them ( $p>0.1$ conjunctival cultures, $p>0.1$ lid margin cultures, Fisher's Exact probability test).
\end{abstract}

A preparation containing trimethoprim and polymyxin $B$ sulphate has been shown to be effective in the treatment of surface ocular infections. ${ }^{1,2,3}$ Trimethoprim is known to be active against a wide spectrum of organisms, including Staphylococcus epidermidis, Staphylococcus aureus, Streptococcus pneumoniae, Streptococcus pyogenes, Haemophilus influenzae, Proteus species and Streptococcus viridans, ${ }^{4,5}$ and polymyxin B sulphate has a spectrum of activity which includes Pseudomonas species. ${ }^{6}$ In addition, synergy against Gram negative organisms has been demonstrated when the two antibiotics are used in combination. ${ }^{7}$ Chloramphenicol preparations are widely used in the topical treatment of bacterial infections of the eye.

It has been demonstrated that topically administered chloramphenicol has no significant effect on ocular flora when administered for a short period ( 24 hours) prior to surgery. ${ }^{8,9}$ Antibiotics which have been

From: Nuffield Laboratory of Ophthalmology, Walton Street, Oxford, ${ }^{1}$ Department of Microbiology, John Radcliffe Hospital, Oxford ${ }^{2}$ and The Wellcome Research Laboratories, Beckenham, Kent. ${ }^{3}$

Correspondence to: J. R. Gibson, Head, Dermatology Section, The Wellcome Research Laboratories, Langley Court, Beckenham, Kent BR3 3BS 
shown to reduce organisms present in the conjunctivae and lid margins when administered for short periods prior to. surgery are gentamicin $^{8}$ and a combination of penicillin and streptomycin. ${ }^{9} \mathrm{We}$ considered it useful to compare the effect of trimethoprim-polymyxin B sulphate ophthalmic ointment with chloramphenicol ophthalmic ointment on organisms present in the conjunctivae and lid margins when administered for a prolonged period to both the operated and unoperated eyes of patients undergoing cataract surgery.

\section{Materials and Methods \\ Recruitment of patients}

Fifty patients undergoing cataract surgery either with or without an intraocular lens implant were recruited consecutively into this study and randomly divided into two treatment groups. Patients were excluded from the study if they had known allergic hypersensitivity to any of the constituents of the test medications, and if they had taken topical or systemic antibacterial agents in the two weeks prior to surgery. One patient in the trimethoprim-polymyxin B sulphate group did not return for her final assessment on day 14 , and therefore clinical and bacteriological assessments were not completed for this patient.

\section{Clinical assessment}

A clinical assessment was made of the appearance of the conjunctivae and lid margins on the pre-operative day (day -1$)$, on the first post-operative day (day 1), on the day the patient was discharged from hospital (day D) and on the fourteenth post-operative day (day 14). The following criteria were assessed on a scale of $0-4(0=$ none, $4=$ severe): conjunctival hyperaemia, presence of discharge, eyelid crusts and reactions around sutures.

\section{Culture technique and bacteriological assess-} ment

Swabs were obtained from each patient on the pre-operative day (day -1 ), the day of surgery (day 0), day after surgery (day 1 ), day of discharge from hospital (day D) and on the fourteenth post-operative day (day 14). The lower conjunctival fornix of each eye was separately swabbed with a cottontipped pernasal swab, and in addition, using one swab for each eye, the upper and lower lid margins of each eye were swabbed. All swabs were placed in Stuart's transport medium. Each swab was plated out in a standardised manner on blood agar and chocolate agar and the swab was then placed in a glucose broth. The blood and chocolate agar plates were incubated in $\mathrm{CO}_{2}$ at $37^{\circ} \mathrm{C}$. The glucose broth was incubated at $37^{\circ} \mathrm{C}$ and subsequently subcultured onto blood agar and chocolate agar. Any quantity of growth was recorded as a positive culture. Separate strains of organisms were counted as separate positive cultures.

\section{Antibiotic administration}

Patients were allocated treatment with an ointment containing either trimethoprim $5 \mathrm{mg} / \mathrm{g}$ and polymyxin B sulphate 10,000 units/g or chloramphenicol $1 \%$. Each 5 gram tube of ointment was coded in a fully randomised manner and two tubes were provided for each patient. The initial swab of the conjunctivae and the lid margins was taken prior to treatment. The dosage regime involved the administration of ointment four times on the pre-operative day, once in the morning before the operation and twice daily until the fourteenth post-operative day.

\section{Surgery}

Intra-ocular surgery comprised either extracapsular surgery and insertion of a posterior chamber intraocular lens or intracapsular surgery without an implant. As it was our usual practice to give a subconjunctival injection containing gentamicin sulphate $20 \mathrm{mg}$ and betamethasone sodium phosphate $4 \mathrm{mg}$ following introduction of an implant, this was administered to every operated eye. In addition to the allocated antibiotic, the postoperative treatment comprised guttae betamethasone sodium phosphate $0.1 \%$ four times daily, guttae cyclopentolate hydrochloride $1 \%$ four times daily and oculentum betamethasone sodium phosphate $0.1 \%$ nocte to the operated eye.

\section{Results}

Table I details bacteria isolated from con- 
junctivae and lid margins pre-treatment. One patient in the trimethoprim-polymyxin B sulphate group grew both Staphylococcus epidermidis and diphtheroids from the conjunctiva of one eye and one patient in the chloramphenicol group grew both Staphylococcus epidermidis and diphtheroids from the lid margin of one eye. It should be noted that during the study period other types of organisms were occasionally cultured including Streptococcus viridans, Coliforms, Staphylococcus arueus, group B Streptococcus and Streptococcus faecalis.

Table II summarises the number of patients with positive cultures obtained from the operated and unoperated eye during the study period. The Sign test was used to

Table I. Bacteria isolated on the pre-operative day

\begin{tabular}{lcrrr}
\hline & \multicolumn{2}{c}{ Conjunctivae $^{+}$} & \multicolumn{2}{c}{ Lid Margins $^{+}$} \\
\hline & Number & $\%$ & Number & $\%$ \\
Staphylococcus epidermidis & 13 & 13 & 26 & 26 \\
Diphtheroids & 2 & 2 & 5 & 5 \\
Proteus species & 1 & 1 & 1 & 1 \\
No growth & 85 & 85 & 69 & 69 \\
\hline
\end{tabular}

+ The number of eyes swabbed $=100$, the number of patients involved $=50$.

One patient in the trimethoprim-polymyxin B sulphate group grew both Staphylococcus epidermidis and diphtheroids from the conjunctiva of one eye, and one patient in the chloramphenicol group grew both Staphylococcus epidermidis and diphtheroids from the lid margin of one eye (thus resulting in a total of 101 for each type of swab).

Table II Comparison of operated with unoperated eye. (Conjunctival and lid margin swabs considered together).

Day -1 Day 0 Day $1 \quad$ Day D Day 14

Trimethoprim-polymyxin

B sulphate group $(n=25)$

Number of patients with

organisms cultured from:

neither eye

operated eye only

unoperated eye only

both eyes

Chloramphenicol group $(n=25)$

Number of patients with

organisms cultured from:

neither eye

operated eye only

unoperated eye only

both eyes

9
2
6
8

13
4
4
4

$\begin{array}{rrr}14 & 16 & 11^{*} \\ 3 & 3 & 2^{*} \\ 6 & 3 & 6^{*} \\ 2 & 3 & 5^{*}\end{array}$

$1 *$

$6^{*}$

$5^{*}$ 
examine differences between the operated and the unoperated eye, and results of the statistical analysis revealed no significant differences in either treatment group ( $p>0.1)$. It was therefore assumed that the surgery and subconjunctival antibiotics had no definite effect on the presence of organisms in the eye, and thus, for the purpose of subsequent analyses no distinction was made between the operated and the unoperated eye.

Table III details the number of patients with positive cultures obtained from the conjunctivae and lid margins during the study period. Scores for conjunctivae and lid margins were obtained by noting a positive culture in either the operated or unoperated eye for each patient. In addition, the conjunctivae and lid margins were considered together for each patient and a score was obtained by noting a positive culture in either the lid margin or conjunctiva of either the operated or unoperated eye.

Table IV details the changes in bacteriological status of the conjunctivae and lid margins of each patient from pre-treatment to post-surgery. Scores were obtained for both conjunctivae and lid margins by dividing patients into the following four groups:

(1) patients who had organisms present both pre-treatment and post-surgery,

(2) patients who had organisms present pretreatment but not post-surgery,
(3) patients who had no organisms present pre-treatment, but who developed organisms post-surgery,

(4) patients who had no organisms either pre-treatment or post-surgery.

In addition, the conjunctivae and lid margins were considered together as before.

The effect of the antibiotics on organisms present in conjunctivae and lid margins and a comparison of the effect of the two preparations was made from an examination of the patients who had organisms present pretreatment but not post-surgery, and the patients who had no organisms present pretreatment but who developed organisms post-surgery (columns 2 and 3 in Table IV). The results can be summarised as follows:

(i) Trimethoprim-polymyxin $B$ sulphate group

Organisms were lost from the conjunctivae of 6 patients and emerged in 5 patients. Organisms were lost from the lid margins of 4 patients and emerged in 5 patients. When the conjunctivae and lid margins were considered together organisms were lost from 5 patients and emerged in 5 patients. The effect of the antibiotic on the number of patients with positive cultures was not significant ( $\mathrm{p}>0.1$, Sign test).

Table III Number of patients with positive cultures obtained from conjunctival and lid margin swabs.

\begin{tabular}{|c|c|c|c|c|c|}
\hline & Day -1 & Day 0 & Day 1 & Day $D$ & Day 14 \\
\hline \multicolumn{6}{|l|}{$\begin{array}{l}\text { Trimethoprim-polymyxin } \\
\text { B sulphate group }(n=25)\end{array}$} \\
\hline Conjunctivae & 7 & 4 & 3 & 3 & $5^{*}$ \\
\hline Lid margins & 13 & 12 & 9 & 6 & $12^{*}$ \\
\hline $\begin{array}{l}\text { Conjunctivae and lid margins } \\
\text { considered together }\end{array}$ & 16 & 12 & 11 & 9 & $13^{*}$ \\
\hline \multicolumn{6}{|l|}{ Chloramphenicol group $(n=25)$} \\
\hline Conjunctivae & 5 & 6 & 7 & 8 & 2 \\
\hline Lid margins & 11 & 12 & 7 & 8 & 10 \\
\hline $\begin{array}{l}\text { Conjunctivae and lid margins } \\
\text { considered together }\end{array}$ & 14 & 16 & 11 & 14 & 10 \\
\hline
\end{tabular}

${ }^{*} \mathrm{n}=24$ 
(ii) Chloramphenicol group

Organisms were lost from the conjunctiva of 1 patient and emerged from the conjunctivae in 6 patients. Organisms were lost from the lid margins of 6 patients and emerged in 10 patients. When the conjunctivae and lid margins were considered together, organisms were lost from 4 patients and emerged in 9 patients. The effect of the antibiotic on the number of patients with positive cultures was not significant $(\mathrm{p}>0.1$, Sign test).

Using data from the same columns, a comparison of the effect of the two preparations was made. No significant difference was found between the two treatments ( $p>0.1$ in each case, Fisher's Exact test).

The results of the clinical assessment of the appearance of conjunctivae and lid margins between the two treatment groups were analysed using Fisher's Exact test. There was no significant difference between the scores obtained in the trimethoprim-polymyxin B sulphate treated group and the chloramphenicol treated group on any day $(\mathrm{p}>0.1)$. However, it should be appreciated that both preparations were well tolerated and that no clinically apparent infections occurred during the study period.

\section{Comment}

The results indicate that the number of organisms present in conjunctivae and lid margins appeared to remain relatively unaffected by the use of either trimethoprimpolymyxin B sulphate ophthalmic ointment or chloramphenicol ophthalmic ointment for sixteen days, although there was a slight trend for trimethoprim-polymyxin B sulphate to perform better than chloramphenicol in reducing the number of positive cultures. It is impossible to comment from our data on whether, despite the lack of overall effect of the two treatments on the ocular flora, they may have played a role in preventing clinically apparent infections, as the study was not designed for this purpose.

Table IV Changes in bacteriological status from pre-treatment to post-surgery.

\begin{tabular}{rrrrrr}
\hline Before & + Growth & + Growth & - Growth & - Growth \\
After & + Growth & - Growth & + Growth & - Growth \\
\hline
\end{tabular}

Trimethoprim-polymyxin

$B$ sulphate group $(n=25)$

Conjunctivae

Lid margins

Conjunctivae and lid margins

considered together

\section{Chloramphenicol group $(n=25)$}

Conjunctivae

Lid margins

Conjunctivae and lid margins

considered together

$\begin{array}{rrr}6 & 5 & 13 \\ 4 & 5 & 7 \\ 5 & 5 & 4\end{array}$

7

Key

+ Growth Organisms present both

+ Growth pre-treatment and on day of discharge or day 14 (post-surgery)

+ Growth Organisms present pre-

- Growth treatment but no organisms present on either day of discharge or day 14 (post-surgery)

- Growth Otganisms were not

+ Growth present pre-treatment but developed by day of discharge or day 14 (post-surgery)

- Growth No growth of organisms

- Growth pre-treatment or on day of discharge or day 14 (post-surgery) 


\section{References}

${ }^{1}$ Genée E, Schlechtweg C, Bauerreiss P, Gibson JR. Trimethoprim-polymyxin eye drops versus neomycin-polymyxin-gramicidin eye drops in the treatment of presumptive bacterial conjunctivitis - a double-blind study. Ophthalmologica 1982; 184: 92-6.

2 van Rensburg SFJ, Gibson JR, Harvey SG, Burke CA. Trimethoprim-polymyxin ophthalmic solution versus chloramphenicol ophthalmic solution in the treatment of bacterial conjunctivitis. Pharmatherapeutica 1982; 3: 274-7.

${ }^{3}$ Gibson JR. Trimethoprim-polymyxin B ophthalmic solution in the treatment of presumptive bacterial conjunctivitis - a multicentre trial of its efficacy versus neomycin-polymyxin B gramicidin and chloramphenicol ophthalmic solutions. J Antimicrob Chemother 1983; 11: 217-21.

${ }^{4}$ Gleckman R, Blagg N, Joubert DW. Trimethoprim: Mechanisms of action, antimicrobial activity, bacterial resistance, pharmacokine- tics, adverse reactions and therapeutic indications. Pharmacotherapy 1981; 1: 14-20.

${ }^{5}$ Bushby SRM and Hitchings GH. Trimethoprim, a sulphonamide potentiator. Br J Pharmacol Chemother 1968; 33: 72-90.

${ }^{6}$ Storm DR, Rosenthal KS, Swanson PE. Polymyxin and related peptide antibiotics. Annu Rev Biochem 1977; 46: 723-63.

${ }^{7}$ Rosenblatt JE and Stewart PR. Combined activity of sulfamethoxazole, trimethoprim and polymyxin B against Gram-negative bacilli. Antimicrob Agents Chemother 1974; 6: 84-92.

${ }^{8}$ Fahmy JA. Bacterial flora in relation to cataract extraction. V Effects of topical antibiotics on the pre-operative conjunctival flora. Acta Ophthalmol 1980; 58: 567-75.

${ }^{9}$ Locatcher-Khorazo D. The effect on the ocular bacterial flora of local treatment with chloromycetin (chloramphenicol), terramycin or penicillin-streptomycin ophthalmic ointments in pre-operative cataract cases and miscellaneous infections. Am J Ophthalmol 1953; 36: $475-9$. 